\title{
Microseparation and Analysis of the Contents of Single Cells Reveal the Intracellular Fate of a TAT-Conjugated Inhibitor
}

\author{
A. P. Manceur, R.B. Brown, J. Audet
}

Institute of Biomaterials and Biomedical Engineering (IBBME), University of Toronto, CANADA, M5S 3G9

Terrence Donnelly Centre for Cellular \& Biomolecular Research (DCCBR), University of Toronto, CANADA, M5S 3E1

Several recent studies have demonstrated the potential of HIV-transactivator of transcription (TAT)mediated delivery of peptides and proteins for various applications in cellular engineering and molecular therapy. In order to improve the clinical utility of this approach, it is necessary to clearly identify the factors limiting the bioactivity of the cargoes delivered. We have used single-cell capillary electrophoresis (CE) in combination with conventional CE and mass spectrometry (MS) to examine the intracellular fate of a TAT-conjugated inhibitor of glycogen synthase kinase-3 (GSK-3). TAT-eIF2B is composed of a 16 amino acid peptide sequence derived from the eukaryotic initiation factor $2 \mathrm{~B}$ which was fluorescently labeled with 5-FAM and linked to TAT(47-57) via two cysteines to form a detachable disulfide bond [1]. The activity of TAT-eIF2B on the GSK-3 signaling pathway was demonstrated using a luciferase reporter assay [1].

We have first optimized the bioanalytical parameters for the single-cell (CE)-laser induced fluorescence assay to resolve and detect eIF2B and phospho-eIF2B when loaded in subphysiological concentration (nM range) in cells. Single-cell CE was performed on a custom-made CE system with laser induced fluorescence (LIF) detection mounted on an inverted microscope equipped with a pulsed laser for cell sampling, as previously described [2]. We were able to detect in single cells the proteolytic cleavage of the inhibitor and directly assess the impact of several D-amino acid substitutions on the resistance of the inhibitor to intracellular proteases. However, it was not possible to observe the phosphorylation of the peptide in live cells. Interestingly, when lysates prepared in the presence of high concentrations of a protease inhibitor cocktail were analysed by conventional CE, it was possible to detect the phosphorylated form of the peptide, suggesting that the rapid cleavage of TAT-eIF2B prevents its phosphorylation in live cells.

We were next interested in determining which site(s) of TAT-eIF2B were cleaved by peptidase(s) since this would provide useful information to modify the peptide at specific amino acid position(s) to increase its intracellular half-life. This was achieved using $\mathrm{CE}$ in association with liquid chromatography (LC)-MS. We first obtained a single-cell CE electropherogram of TAT-eIF2B loaded in cells showing the different proteolytic fragments derived from TAT-eIF2B (Figure 2A,B). Then a scaled up preparation was obtained by lysing $\sim 10^{5} \mathrm{TF}-1$ cells loaded with TAT-eIF2B. The peptide fragments were immunopurified by targeting the fluorescein tag (5-FAM). A sample of the immunopurified fragment preparation was analysed by $\mathrm{CE}$ to determine how closely it matched the single-cell electropherogram (Figure $2 \mathrm{~B}$ and $2 \mathrm{C}$ ). As expected, the electropherogram obtained from the lysate has a few additional peaks compared with the single cell version. However, it was sufficiently similar to attempt the identification of the peptide fragments using LC-MS. The LC-MS 
analysis revealed four potential cleavage sites in eIF2B which can now be modified to increase its resistance to proteases and, therefore, its bioactivity.

In summary, single-cell $\mathrm{CE}$ is a powerful tool to monitor the action of intracellular enzymes on exogenous peptide substrates and inhibitors delivered using TAT. In association with MS, CE can facilitate the identification of amino acid sequence modifications that aim at increasing the intracellular half-lives of cargoes delivered using TAT.
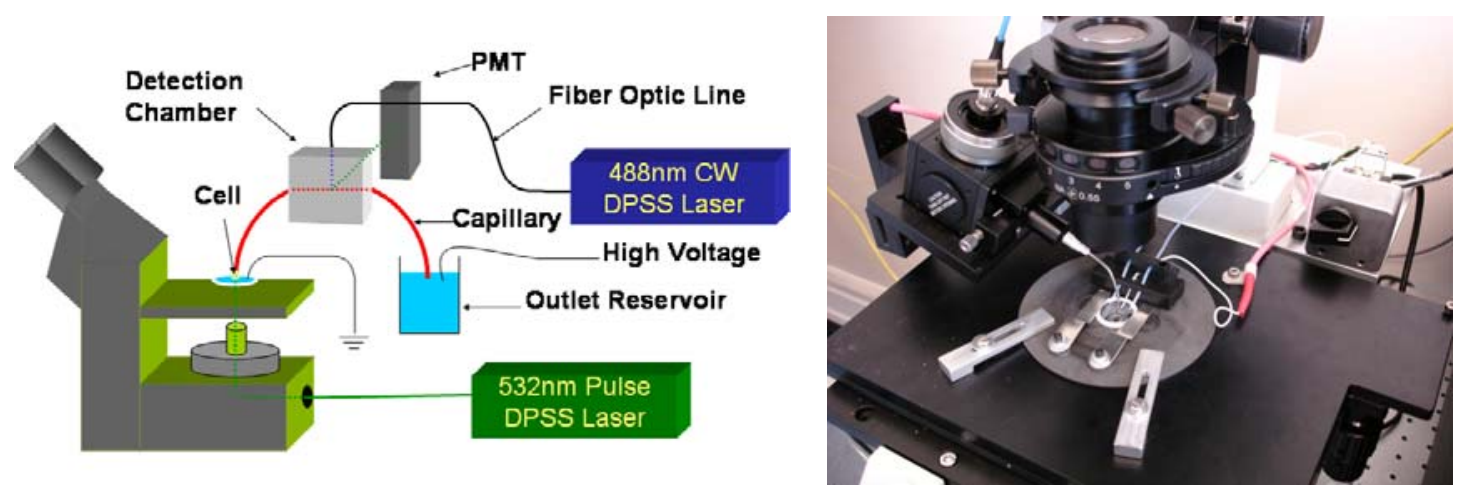

FIG 1. Single-cell CE system. CE was performed with 30 micron internal diameter bare silica capillaries externally coated with polyimide (Polymicro Technologies, Phoenix, AZ, USA) cut to a length of $38 \mathrm{~cm}$ with a detection window created at $12 \mathrm{~cm}$ from the inlet. A voltage of $9 \mathrm{kV}$ on negative polarity in the outlet reservoir was used for electrophoretic separations. Electrophoresis run buffer is physiological buffer at $\mathrm{pH}$ of 7.4.

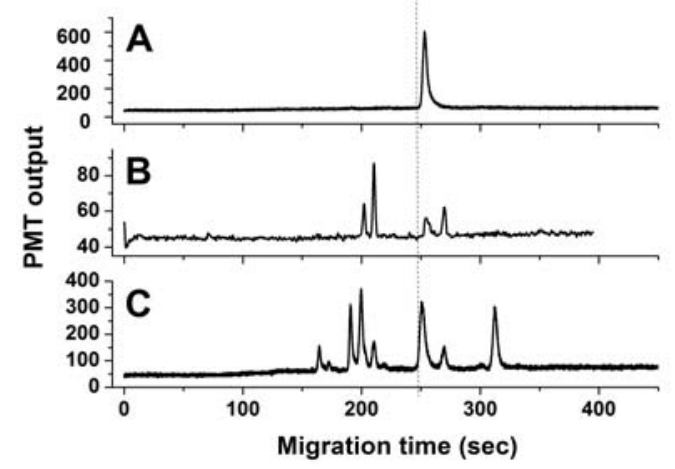

FIG 2. Capillary electrophoresis analyses of TAT-eIF2B. A) Migration standard (TAT-eIF2B); B) Single TF-1 cell loaded with TAT-eIF2B; C) Lysate prepared from TF-1 cells loaded with TATeIF2B after immunoprecipitation with an anti-FITC antibody.

References

[1] A. P. Manceur et al., Molec. Ther. 17 (2009) 500.

[2] R.B. Brown et J. Audet, Cytometry. 71A (2007) 882. 